\title{
Poblaciones expuestas a agrotóxicos: autoorganización ciudadana en la defensa de la vida y la salud, Ciudad de Córdoba, Argentina
}

\section{$\mid{ }^{1}$ Mauricio Berger, ${ }^{2}$ Francisco Ortega |}

Resumo: El artículo presenta un adelanto de nuestro trabajo de investigación doctoral basado en la acción política del grupo de mujeres autodenominado "Madres de Barrio Ituzaingó Anexo", de la ciudad de Córdoba, Argentina. Desde fines de 2001, las integrantes de este colectivo se disponen a luchar por la vida, la salud y el ambiente ante un problema ambiental, que genera más de 200 casos entre enfermedades y muertes, en una población de 5.000 habitantes del barrio, principalmente a causa de las fumigaciones con agrotóxicos de los campos de soja colindantes a las viviendas. Las modalidades de autoorganización desplegadas por "las Madres" frente a la vulneración de derechos y ante la ineficacia y a veces abandono del estado en la protección de los afectados por la contaminación, hacen de esta práctica ciudadana un caso ejemplar para pensar la actualidad de las luchas por derechos. El artículo se estructura en tres secciones. En primer lugar, daremos cuenta de las elecciones metodológicas y conceptuales que enmarcan nuestras reflexiones: las contribuciones del pragmatismo (Dewey y Joas), el giro narrativo (Ricoeur) y discusiones contemporáneas en biopolítica (Agamben, Hardt y Negri, Virno). En segundo lugar, ponemos en común la lucha de las Madres de Barrio Ituzaingó Anexo y sus contextos. En tercer lugar describimos algunas escenas de la lucha de las Madres contra la fumigación con agrotóxicos, para lo cual introducimos tres nociones para pensar la autoorganización ciudadana: cuerpo, experiencia y narración. Las mismas serán consideradas aspectos clave de la práctica política en los que se juega el sentido de la misma como acción, como poder de hacer y decir de la ciudadanía que actúa en autodefensa. A través del ejercicio de sus capacidades afectivas, cognitivas y lingüísticas, estas ciudadanas generan aprendizajes, saberes prácticos y logros en la defensa de sus derechos, tarea no exenta de problemas y obstáculos en sus reclamos de justicia para sus demandas.

\author{
${ }^{1}$ Doutorando em Ciências \\ Sociais, Universidade de \\ Buenos Aires, Argentina; \\ bolsista do Consejo Nacional de \\ Ciencia y Técnica (CONICET); \\ integrante da equipe de \\ pesquisadores do "Proyecto \\ Ciudadanía" e docente de \\ Política y Comunicación, ambos \\ na Universidad Nacional de \\ Córdoba, Argentina. Endereço \\ eletrônico: mauricio.berger@ \\ gmail.com \\ 2 Professor adjunto do Instituto \\ de Medicina Social da UERJ. \\ Coordenador do Programa de \\ Estudos da Ação e do Sujeito \\ (PEPAS), IMS-UERJ. Endereço \\ eletrônico: fjortega2@gmail. \\ com
}

Recibido: 04/02/2009. Aprobado: 15/12/2009.

> Palavras-chave: acción; derechos; salud; ambiente; agrotóxicos. 


\section{Introducción $^{1}$}

El presente artículo presenta un adelanto de nuestro trabajo de investigación doctoral, en el que partimos del análisis de un caso particular, la acción del colectivo de mujeres autodenominado "Madres de Barrio Ituzaingó Anexo", de la ciudad de Córdoba, para pensar la actualidad de las luchas por los derechos en cuestiones ambientales y de salud en Argentina. A finales de 2001, las integrantes de este colectivo se disponen a luchar por la vida, la salud y el ambiente ante un problema ambiental, que genera enfermedades y muertes numerosas en una población de 5.000 habitantes, de este barrio en la periferia industrial de la ciudad de Córdoba. La causa principal de la contaminación es la actividad de fumigación con agrotóxicos de los campos de soja colindantes a sus viviendas. Las modalidades de autoorganización por estas mujeres desplegadas frente a la vulneración de derechos y ante la ineficacia y/ o abandono del estado en la protección de los afectados, hacen de la lucha de las Madres un caso ejemplar que configura nuestro interés de investigación.

El artículo se estructura en tres partes: en primer lugar, daremos cuenta de nuestras elecciones metodológicas y conceptuales que enmarcan nuestras reflexiones, a través de las contribuciones del pragmatismo (Dewey y Joas), el giro narrativo (Ricoeur) y discusiones contemporáneas en biopolítica (Agamben, Hardt y Negri, Virno). En segundo lugar, ponemos en común la lucha de las Madres de Barrio Ituzaingó Anexo y los contextos que completan el sentido de la misma. En tercer lugar describimos algunas escenas de la lucha de las Madres contra la fumigación con agrotóxicos. En esta tarea introduciremos tres nociones que a partir de nuestro análisis hemos considerado claves de la autoorganización ciudadana: cuerpo, experiencia y narración. Estos aspectos se anudan en el sentido de la práctica política como acción, como poder de hacer y decir de la ciudadanía que actúan en defensa de sus derechos.

\section{Aspectos metodológicos y teóricos de nuestra investigación}

Algo que nos ha permitido el giro lingüistico en ciencias sociales es una nueva perspectiva para entender que el significado de las palabras y el sentido de las oraciones está dado por su uso en contextos intersubjetivos (PITKIN, 1984). El giro lingüistico permite entonces problematizar la misma construcción de saberes como contrucción de sentido común o conflictivo, a partir de considerar 
el significado como público y compartido por los hablantes en una situación.

Para el analisis de los mismos, la estrategia es trabajar sobre en el marco de redes interconceptuales (NAISHTAT, 2005a) o juegos del lenguaje, siguiendo el aporte de Ludwig Wittgenstein (PITKIN, 1984). En nuestra orientación metodológica la comprensión ha buscado fundarse en lo público de la interacción, de nuestro encuentro entre investigadores y protagonistas de la acción. Superando los distanciamientos sujeto- objeto que suelen caracterizar buena parte del discurso de las ciencias sociales, asumimos la tarea de investigación como forma de cooperar en la generación de sentido de la lucha de las Madres, a través de la reconstrucción de la acción y sus problemas en contextos situados, de nuestra participación en el juego de lenguaje de los derechos.

En una primera etapa establecimos Conversaciones con las integrantes del grupo de Madres. A diferencia de los métodos de la historia oral, que entrelazan los dispositivos de investigación con formas de evaluación cognitivas, emotivas y morales en la producción de verdad, en las Conversaciones el dispositivo es un diálogo que intenta entender-nos con otros acerca de una situación. La interpelación y la interpretación son recíprocas. El Análisis de los marcos o frames (EDER, 1998; GOFFMAN, 2006), como complemento de las Conversaciones, nos ha permitido poner a la vista la trama en/desde la que se piensa, la gramática que enmarca y es recreada por la acción. El foco estuvo puesto en el uso de las palabras con las Madres piensan su práctica, el significado que le asignan y el que obtiene en el contexto de la práctica. Como resultado, se obtienen registros de distintas formas de pensar y actuar la política en el colectivo. En una segunda etapa, la investigación estuvo orientada a la Reconstrucción de la trayectoria de la práctica, desde sus orígenes a la situación actual, tratando de identificar los logros y los problemas o dificultades de la acción en su transcurso, en la interacción con el estado y otros actores. La reconstrucción se dirige entonces a profundizar la comprensión de la complejidad de juegos de lenguaje operantes en la situación, recreando nociones, procedimientos, contextos que definen el sentido de la acción de las Madres. Por último cabe señalar el Análisis documental que nos ha permitido una delimitación del contexto intersubjetivo. El análisis de fuentes periodísticas, documentos oficiales y otros producidos por los protagonistas, nos permitió identificar los posicionamientos en la situación, los discursos y actuaciones que entraban en conflicto, las disputas públicas por la caracterización de la misma. 


\section{La elección de redes conceptuales}

A partir de la reconstrucción que hemos realizado de la lucha de las Madres, nuestro concepto de práctica política recupera en primer lugar su sentido de acción, de ejericio de la autonomía como participación democrática e igualitaria en la construcción de lo público (PITKIN, 1984; ARENDT, 1995, 2001), la naturaleza pública de la política, la política como el campo de acción que surge de la acción concertada, sobre la base del ejercicio ciudadano. Lo que está en el centro de una práctica política es la acción, la formación de un nosotros: qué haremos, quiénes somos, qué queremos. Para profundizar este análisis de la política como acción, en el marco de nuestra tesis doctoral hemos elegido tres redes conceptuales por las perspectivas y definiciones teóricas: biopolítica, pragmatismo y giro narrativo.

En primer lugar de la red conceptual de la biopolítica (FOUCAULT, 1996; 2002; 2006), podemos pensar la relación entre vida y política que se ponen en juego en la defensa de derechos. En particular nos interesa pensar la tensión existente entre la administración estatal de la vida de la población y las formas embrionarias de autogobierno, las prácticas de autoorganización de los afectados. Es decir, la vida como objeto del poder y la vida como resistencia. Dentro de las discusiones contemporáneas en biopolítica, los sujetos políticos son reducidos a vidas desnudas (AGAMBEN, 2001, 2003), despojados de sus derechos, confinados a la supervivencia bajo el estado de excepción en el que el poder soberano decide sobre el valor o disvalor de la vida (ORTEGA, 2004; AGAMBEN, 2001). A esta concepción "negativa” de la biopolítica, como control y dominación, podemos contraponer la posibilidad de una biopolítica "afirmativa", es decir, aquella que recupera la experiencia y la creatividad de las singularidades en la reapropiación de la producción biopolítica de su existencia: cuerpo, afectividad, deseo, creatividad, comunicación (HARDT; NEGRI, 2004; 2001), y en la definición de modalidades de autogobierno, de esferas públicas no estatales frente a la administración estatal (VIRNO, 2003, 2005). Tanto en la biopolítica de signo negativo como en la afirmativa, los cruces entre vida, corporalidad, poder y política hacen de cuerpo y potencia un concepto central para hacer referencia tanto a lo que puede un cuerpo para una política de autodefensa, como veremos en nuestro caso de análisis, como lo que determina la captura, la modulación de esa potencia. 
En cuanto a las contribuciones del pragmatismo, en tanto que filosofía social y política, en la obra de Dewey como referencia central (JOAS, 1990), lo que cuenta en esta perspectiva es la resolución creativa de los problemas de la acción del grupo, problemas de la constitución de un público fundado en una comunidad de acción. La comunicación dirigida a la resolución de problemas de interés colectivo se convierte en una condición esencial del orden social (JOAS, 1990, p. 124). El autogobierno que nos interesa pensar, en esta perspectiva se entiende como la comunicación y la resolución de problemas colectivos, es decir, como autocontrol. La acción no se entiende como la realización de fines preestablecidos, sino que el establecimiento de los mismos no es un acto que tenga lugar fuera de los contextos de la acción. Abordaremos también la relación entre cuerpo y acción, a través de dos conceptos: esquema corporal e intercorporalidad. (JOAS, 1996; WEISS, 1999). También con la noción de experiencia, en tres significados que abrevan del desarrollo que Dewey realizó de la misma: vivencia, aprendizaje, creatividad (DEWEY, 2004; BERNSTEIN, 1996; WEST, 2008).

Por último, el giro narrativo que adoptamos para nuestro análisis nos permitirá ahondar el alcance de la agencia individual y colectiva en la definición de la acción. Aquí la narración y la generación de sentido para la lucha de las Madres son considerados parte de la acción. Con una referencia central en Ricoeur, la noción de identidad narrativa refiere a la construcción de un sí mismo y su presentación a otros sujetos. Se concibe la identidad como algo que se gesta a partir de los lenguajes y relatos disponibles donde se desenvuelve el sujeto (GORLIER, 2002, p. 133). Desde este planteo, el tema que le interesa a Ricoeur es la comprensión de sí, la identidad que el sujeto humano alcanza mediante la función narrativa (RICOEUR, 1999, p. 215).

En síntesis, a través de un trabajo que integra la lectura del caso empírico con las redes conceptuales del pragmatismo, el giro narrativo y la biopolítica, en estas pretendemos adensar el análisis del sentido de la lucha por derechos a partir de la práctica de las Madres. En la tercera parte de nuestro artículo describimos algunas escenas de la lucha de las Madres contra la fumigación con agrotóxicos, introduciendo tres nociones que a partir de nuestro análisis hemos considerado claves de la autoorganización ciudadana: cuerpo, experiencia y narración. Estos aspectos se anudan en el sentido de la práctica política como acción, como poder de hacer y decir de la ciudadanía que actúan en defensa de sus derechos. 
Previamente, y para comprender el sentido y los alcances de las acciones de las poblaciones afectadas por la utilización de agrotóxicos, y de la mayoría de las luchas ambientales que hoy tienen un momento de singular relevancia en Argentina resulta pertinente señalar algunos aspectos contextuales, en la segunda parte de nuestro artículo.

\section{La lucha de las Madres de Barrio Ituzaingó Anexo de la Ciudad de Córdoba, Argentina y sus contextos ${ }^{2}$}

A continuación presentamos dos aspectos contextuales con los cuales podemos entender mejor la práctica política de las Madres de Barrio Ituzaingó Anexo. Por un lado, aquellos que refieren a la producción sojera que utiliza agrotóxicos en Argentina actualmente y la connivencia del Estado en desmedro de los derechos de sus habitantes. Por otro, los acontecimientos políticos del año 2001- la situación política de muchos ciudadanos que se autoorganizan en defensa propia frente a la vulneración de sus derechos- también enmarca la lucha de las Madres.

Por un lado, en lo que refiere a la importancia de la producción sojera en el país, y en particular la provincia de Córdoba como principal productora nacional, con lo que esto implica a nivel de un complejo entramado de intereses económicos e intervención estatal y de sus consecuencias sobre la degradación del medio ambiente y el daño de la salud de las poblaciones como corolario del empleo de agrotóxicos. En un país con una economía principalmente agroindustrial, ubicado como el mayor productor e industrializador de soja del cono sur y tercer exportador mundial, la cotización de la tonelada de soja alcanza precios altísimos en el mercado, por lo cual ya se han destinados más de 16, 6 millones de hectáreas a su cultivo, no sólo desplazando otros tipos de producciones hacia el modelo del monocultivo, sino que también provocando el desmonte de amplias zonas de bosques nativos. La provincia de Córdoba, situada en el centro del país y de la zona pampeana agrícola por excelencia, participa con el 31\% del total de la producción, liderando el primer lugar en el ranking nacional. La producción se basa casi exclusivamente en soja transgénica con tecnología RR, que requiere el empleo de agrotóxicos ya prohibidos o fuertemente regulados por leyes provinciales y nacionales, y controles fitosanitarios. Sin embargo en la práctica, estos agrotóxicos se utilizan indiscriminadamente, bajo el auspicio de grandes multinacionales como Monsanto, Cargill, entre otras. Las poblaciones 
afectadas por el empleo de estos agrotóxicos, y numerosas organizaciones de la sociedad civil, así como distintos movimientos sociales vienen denunciando sistemáticamente las dramáticas consecuencias, que incluyen la desforestación, el desalojo de poblaciones rurales e indígenas, y en particular para lo que nos interesa a los efectos de este artículo, el daño a la salud de las poblaciones y la contaminación de suelo, agua, tierra y aire.

La actividad de fumigación con agrotóxicos no se realiza en ausencia de marcos institucionales y jurídicos que establecen garantías a la salud y al ambiente de los habitantes. Existen normativas establecidas a través de las Constituciones Nacional y Provincial, Ley Nacional de Ambiente, Leyes Provinciales de Ambiente y de Agrotóxicos, que sin embargo no se actualizan. Tras décadas de reforma neoliberal del estado bajo los principios de achicamiento, privatización, descentralización, las instituciones encargadas del control y del poder de policía han sido desmanteladas. Sumado esto al déficit de la representación política en el sistema formal de autoridad, llevó a la ciudadanía a una situación de abandono y desprotección de sus derechos, un hacerse cargo individual y colectivamente de sus situaciones, a asumir la transferencia de responsabilidades públicas como autorresponsabilización. En el escenario del desmontaje del estado bienestarista, las responsabilidades por la salud se delegan en los individuos, exprimiendo su autonomía y agencia (ORTEGA, 2004).

Cabe señalar también que en las situaciones de conflicto ambiental en nuestro país en los últimos años, el estado actúa "positivamente" generando condiciones para explotaciones de diversa índole: se otorgan créditos y exenciones impositivas, se realizan planes de promoción, se construye infraestructura pública para la producción y circulación de las mercancías, etc. Paralelamente el estado actúa por omisión respecto a la generación de condiciones para la realización de ciudadanía. El concepto de ley incluye el de un poder de policía que imponga su cumplimiento (para el caso, policías que impidan la fumigación). El cuidado de la salud pública no dispone de infraestructura (hospitales, dispensarios) ni procedimientos administrativos, ni protocolos de intervención profesional para casos de las enfermedades y malformaciones, plaguicidas en la sangre de niños, etc. El estado no ofrece garantías, es decir, no instituye acciones destinadas a sostener de modo permanente los derechos reconocidos constitucionalmente. La omisión se presenta así como un dispositivo de despojamiento de identidad ciudadana, entendiendo la misma como el derecho a tener derechos (CARRIZO; BERGER, 2009). 
Otro aspecto contextual que nos interesa poner de relieve para el análisis de ejercicio y defensa de derechos ambientales, es la configuración de las prácticas políticas a partir del acontecimiento del 19 y 20 de diciembre de 2001 en Argentina. El mismo marca la crisis política e institucional del estado democrático de derecho, y que se expresa en una multitud de experiencias de deliberación pública y horizontal en asambleas vecinales, fábricas ocupadas y gestionadas por sus trabajadores, redes de trueque, movimientos de trabajadores desocupados, y otras más en las que se juega el ejercicio de la autonomía política frente a una clase dirigente desacreditada e instituciones vaciadas de sentido, al punto de llegar a hacer caer el gobierno constitucional de Fernando de la Rúa.

Frente a la transferencia ilimitada de responsabilidades públicas a sectores de la sociedad, y las consecuencias del desmantelamiento neoliberal del estado, la ciudadanía se autoorganiza en autodefensa. En esta situación, la diversidad de expresiones políticas han tenido distintas orientaciones, desde la invocación y revocatoria de la institucionalidad del estado democrático de derecho, sostiene lo que tiene de público la política en un contexto de crisis de efectividad y legitimidad del sistema político, en tanto garante de los derechos y principios de política instituidos en la Constitución y las leyes. (CARRIZO; BERGER, 2009). En ese sentido las Madres de Barrio Ituzaingó se inscriben también en este acontecimiento político, según relatan:

Empezamos como cualquier ciudadano que sabe que tiene derecho a pedir al gobierno, [...] que estaban violando los derechos. Uno empieza como se empezó en la Plaza de Mayo el 20 de diciembre de 2001; la gente sabía que tenía que salir a la calle a reclamar y pasó eso... Había algo que estaba mal...

En el caso de las Madres, el ejercicio del derecho comienza a fines del año 2001, con la petición a las autoridades para esclarecer las causas de la contaminación y sus efectos en la salud de la población entre lo que se encuentran derrames de PCB de los transformadores de energía eléctrica, residuos industriales de fábricas automotrices, y principalmente la fumigación de los campos de soja aledaños a las viviendas con agrotóxicos, de forma ilegal, ya que se violan los requisitos establecidos por ley para dicha actividad.

La ineficacia de las instituciones y organizaciones públicas, lleva a este colectivo de mujeres a conformar una red de perjudicados sobre la base de su experiencia común. La acción del grupo de Madres de Barrio Ituzaingó, los vecinos del barrio y una red de actores comprometidos instala el tema públicamente, dirige el 
reclamo hacia instituciones puntuales por las funciones que éstas deben cumplir.

En lugar de la auto-responsabilización, la práctica política responsabiliza en primera instancia a los funcionarios comprometidos, a los que les exige una acción protectora. En segundo lugar, con la crítica de la ciudadanía hacia los criterios y efectividad de la intervención, se realiza una segunda responsabilización, tanto institucional como pública: el cuestionamiento a los resultados de la actuación. La invocación de las autoridades públicas estatales es en el mismo acto una revocación de la legitimidad de su mandato, una crítica destituyente ante la ineficacia e indolencia de su intervención en resguardo de los derechos.

Los afectados por un daño o conflicto toman medidas para salvaguardar sus intereses, instaurando acuerdos mutuos y otros medios para dirimir los problemas, definiendo los rasgos embrionarios que definen a un Estado, sus organismos y sus funcionarios (DEWEY, 2004, p. 66).

Vemos aquí la tensión entre una biopolítica afirmativa y otra negativa a la que hacíamos referencia previamente. En la defensa de la vida y la salud, entran en tensión la práctica ciudadana con las estrategias de dominio de la administración estatal, que procuran reapropiarse de lo "auto" de la práctica ciudadana, ya no tan solo en clave de transferencia de responsabilidades públicas por el desmantelamiento de la estructura bienestarista del estado, sino ahora también respecto de la regulación de la existencia política de ciudadanos autoorganizados.

En el caso de las Madres de B. Ituzaingó, a la situación originaria de vulneración de derechos a causa de la contaminación ambiental, sus efectos en las enfermedades y muertes, en la destrucción del ambiente, se añade otra situación de vulneración de derechos, ahora políticos. El derecho a la vida política, la constitución de un colectivo, la participación efectiva de los afectados en la vida pública, en la resolución de un problema público también se ven obstaculizados o privados de un ejercicio efectivo, de reconocimiento por parte del Estado. Estrategias de dominio por parte de actores estatales apuntan a desarticular y desmotivar los colectivos que resisten, logrando como efecto la pérdida o parálisis de la capacidad de acción de la ciudadanía, de lo cual el colectivo de las Madres constituye un caso testigo. Mecanismos con los que la administración estatal controla, regula, modula no sólo la vida de la población sino la existencia política de la ciudadanía.

En el siguiente apartado describiremos algunas escenas del recorrido exhaustivo de las Madres por las instituciones del Estado, reuniones con funcionarios públicos 
en búsqueda de soluciones para los problemas de contaminación. Como veremos en la descripción, las respuestas de parte del estado se definen entre la ineficacia, el desconocimiento de la problemática del barrio o intervenciones tecnocráticas que diluyen el carácter de estado democrático de derecho. Así también los ataques de parte de funcionarios estatales que ha recibido el grupo de Madres, y que produce la desmotivación y desmovilización del colectivo. A casi diez años de comenzada la lucha de las Madres, la falta de soluciones y la tentativa oficial de ocultar la problemática agudiza la situación de injusticia que padecen los habitantes de Barrio Ituzaingó Anexo.

A continuación presentaremos brevemente algunas escenas en las que podemos describir la práctica de autoorganización ciudadana en defensa de la vida y el ambiente. La situación de ciudadanos que recurren a sus propias fuerzas y capacidades afectivas, intelectuales, cognitivas, comunicativas ante la falta de actuación del estado, generando vivencias y aprendizajes de acción, de interacción, de un entramado intersubjetivo para la defensa de los derechos. Ciudadanos autoorganizados que son también capaces de generar lecturas, reflexiones sobre la propia marcha de la acción, producto de lo cual se afirman o revisan identidades, relatos, historias, sentidos. Para desarrollar este análisis proponemos tres nociones claves, que presentaremos resumidamente a continuación: cuerpo, experiencia y narración.

\section{Cuerpo, experiencia y narración: nociones claves de una práctica de autoorganización en la defensa de la vida y la salud como derechos}

Producto de nuestra interacción con las Madres de Barrio Ituzaingó Anexo y de nuestra elección de las redes conceptuales del pragmatismo, la biopolítica y el giro narrativo que presentamos previamente, hemos identificado las nociones de cuerpo, experiencia y narración como claves de lectura para comprender una práctica de autoorganización ciudadana en la defensa de derechos, en situaciones de vulneración de los mismos y de faltas y fallas graves en la actuación estatal para el reconocimiento y garantía de los derechos vulnerados. Ante la falta o los déficits de protección de parte del estado, los ciudadanos que se autoorganizan en defensa propia cuentan con sus propios cuerpos, la experiencia y la narración de su lucha. 


\section{Cuerpo}

En lugar de resolver estos problemas de forma aislada y como si fuera una patología individual, las Madres y otros vecinos del barrio sospechan e identifican algunos factores de contaminación en el barrio: los transformadores de la energía eléctrica, las fumigaciones de los campos de soja colindantes a las viviendas, el suministro de agua. Se dan cuenta que algo las está dañando, desde la percepción y la afección por el padecimiento de sus familiares. Entre la percepción de la situación y los sentimientos que les genera, entre la preocupación, la angustia, el dolor, comienza la movilización para buscar en primer lugar el esclarecimiento de los problemas y la intervención de las autoridades en su solución.

A nosotras también nos movilizó mucho el dolor... vos sabés que tu vecino tiene un hijo enfermo, o tiene un nieto y una sabe el dolor que siente, sabe lo que siente una mamá con un hijo con un cáncer o una leucemia, una sabe y sabemos hasta dónde puede llegar esa criatura, eso a nosotros nos moviliza y nos movilizó siempre...

¿Y sabés de dónde sacás más fuerza? Cuando te quitan un hijo no te lo devuelven, te quitan todo, porque también perdí mi marido. Entonces ésa es la fuerza que te da para seguir. Porque si perdiste un hijo no querés perder otro, porque si perdiste un marido no querés perder otro ser querido. Porque no te van a devolver la vida de tu hijo ni te van a dar la solución a los problemas. Y las soluciones no te vuelven y tenés que andar luchando cuando un remedio tendría que estar ahí.

Uno de los aspectos de las prácticas de autoorganización es la movilización ciudadana a partir de los sentimientos de dolor tanto como de defensa de la vida, una voluntad y una fuerza de existir que pone a los cuerpos en movimiento. En el caso de las Madres, no hay una toma de conciencia de la situación como si fuera una adquisición racional de ideas sobre el problema, sino que hay una afectación de los cuerpos, sentimientos y sensaciones a partir de lo cual se origina la acción.

Como podremos apreciar en la descripción de la experiencia de las Madres, la situación que enfrenta la ciudadanía afectada por un problema ambiental, con efectos sobre su salud, sobre sus vidas, es de soledad ante el daño, no hay estructuras institucionales que actúen en la prevención, protección o remediación del daño:

Ellos, con las fumigaciones, nos han fumigado con avionetas, no les importó la gente, nadie fue capaz de decir: No, hay un barrio cerca... No, si no salía la gente, si no salíamos nosotros a pelear, esto todavía lo seguirían haciendo. Se frenaron gracias a la pelea. La organización surgió porque tuvimos que defendernos de algo que nos estaba dañando. 

tenemos que hacer eso, tratar de defendernos solas con lo que tenemos, que son pocos recursos.

Defenderse solas con los pocos recursos que tienen: sus personas, sus propios cuerpos en primer lugar, las facultades emotivas, afectivas, cognitivas que ponen en juego para la autoorganización. Estas facultades no son algo objetivo que tiene lugar en el cuerpo sino una genérica capacidad de actuación que sucede entre los cuerpos (VIRNO, 2005).

Nuestra percepción del mundo está estructurada por nuestras capacidades para y experiencias de acción, de allí que el establecimiento de los fines de la acción se puede explicar haciendo referencia a la corporalidad de los actores, y a las metas como algo que surge de la acción misma (JOAS, 1996).

Son las capacidades, hábitos y formas corporales de relacionarnos con el ambiente las que constituyen el background para cualquier establecimiento consciente de objetivos, en otras palabras, de la intencionalidad (JOAS, 1996, p. 158).

La noción de esquema corporal que elabora Merleau Ponty (JOAS, 1996; WEISS, 1999) nos permite entonces pensar el cuerpo no como objeto, como un conjunto de órganos biológicos, sino en tanto que comportamiento, como esquema para la acción. A los fines de nuestra investigación, nos interesa pensar el esquema corporal no sólo como acción motora sino respecto de la defensa de derechos como acción política.

No hablamos de esquemas corporales individuales. Todo este esfuerzo que acusan los cuerpos se sostiene también por el sentimiento del otro próximo- prójimo. En el transcurso de su acción, las Madres no solo luchan por el esclarecimiento de las causas de enfermedades en el barrio y por el cese del daño, sino que también han desplegado formas de solidaridad y de regeneración de los vínculos en el barrio. Contra las descalificaciones y ataques que reciben de parte de las autoridades del centro de participación vecinal, las Madres han organizado distintas actividades para reafirmar su presencia en el barrio, por ejemplo un gran festejo colectivo del día de la Madre. En estos eventos no están las consignas de su lucha contra la contaminación, sino que hay lazo social y afectivo. Vemos en este vínculo un encuentro con la noción de intercorporalidad, también acuñada por Merleau Ponty (JOAS, 1996; WEISS, 1999). La misma refiere a la comunicación 
prelingüística como constitutiva del esquema corporal, la relación del actor con su cuerpo está modelada por estructuras intersubjetivas en las que se juegan los sentimientos de amor y solidaridad hacia el otro. Aquí es cuando podemos pensar la noción de intercorporalidad, tambiéndesde el aporte de Merleau Ponty, como aquella socialidad en la que prima la experiencia corporalmente vivida, la percepción y la empatía, lo sensorial, la sensibilidad, aspectos que son constitutivos también de la acción política.

Junto a estas, otras reflexiones acuden a este problema del cuerpo para la acción. Desde la red conceptual de la biopolítica, el cuerpo es pensando como la potencia de existir y producir, como la capacidad de obrar en relación a los otros cuerpos (HARDT; NEGRI, 2001, 2004; VIRNO, 2003, 2005). La potencia de acción o fuerza de existir aumenta o disminuye puesto que la potencia de otro se añade a la suya o por el contrario, la sustrae, la inmoviliza y la fija. Recuperamos también otro de los conceptos que hacen a la definición de potencia: conatus o apetito. Éste refiere al esfuerzo por experimentar alegría, aumentar la potencia de la acción, imaginar y encontrar lo que es causa de alegría, lo que sustenta y favorece esta causa, y también esfuerzo por evitar la tristeza, imaginar y encontrar lo que destruye la causa de la tristeza. (DELEUZE, 2004, p. 122) Las diversas funciones del conatus son: mecánica (conservar, mantener, perseverar); dinámica (aumentar, favorecer). En la situación de las Madres, el deseo de vivir, el deseo de justicia y de ser escuchados, de que se reconozca el problema, tanto el deseo de no morir aumenta la fuerza de la acción de autodefensa frente a la vulneración de sus derechos. En proporción inversa, los afectos tristes disminuyen la potencia de la acción de las Madres, ocasionando el bloqueo de la acción, por lo cual, la potencia no circula, no genera. La indiferencia y hostilidad de los funcionarios que las atienden, que deniegan el problema, que no responden a sus reclamos provocan sentimientos de angustia, de incertidumbre, de confusión que no alienta la acción sino que le plantea problemas, obstáculos. Se opone como fuerza negativa a la fuerza de existir de las Madres, en tanto cuerpos afectantes que no hacen a la composición y los buenos encuentros sino a la descomposición de la acción en sí. Siguiendo esta propuesta teórica, entendemos por descomposición las situaciones en las que hay desmotivación, desmovilización, desviación en la lucha de las Madres respecto de los fines que orientan su accionar. 


\section{Experiencia}

Las prácticas de autoorganización suelen ser atravesadas por evaluaciones realizadas por los propios actores, en el caso de las Madres, y por la red de actores que se comprometen con ellas. En estas evaluaciones de los logros y problemas de la acción se pone en consideración la racionalidad de la acción, la definición de la estrategia. Mientras que los actores tradicionales de la política (partidos políticos, sindicatos, organismos de Derechos Humanos) no han actuado en el caso como lo han hecho las propias afectadas por la contaminación, sí en cambio realizan evaluaciones de la lucha de las Madres, cuestionándoles su estrategia, la pertinencia de los objetivos, del camino a seguir, de la lectura que hay que realizar de la situación.

Lejos de poder definir con una conciencia racional ajena al contexto de la acción, en la mayoría de las oportunidades las Madres defininen el curso de acción a seguir a partir de sus vivencias. Los objetivos y caminos a recorrer se hacen al andar y no se resuelven externamente al contexto de lucha, institucional, al contexto de interacción con los funcionarios públicos. De este uso también podemos pensar que el involucramiento de las personas en la acción no se da por una conciencia racional o por un acto de concientización respecto del problema sino a partir de vivir un problema, de que pase por el cuerpo como padecimiento, como sentimiento compartido. El carácter vivencial de la experiencia (BERNSTEIN, 1996) comporta una auto-percepción que marca un "entre”, a partir de lo vivido antes y después de la experiencia política. Este "entre” señala lo autotransformador de la experiencia, como las Madres señalan "ya no somos las de antes”.

La experiencia es principalmente un proceso de pasar por algo: un proceso de soportar algo, de sufrimiento y pasión, de afecto, en el sentido literal de estas palabras. El organismo tiene que soportar, que pasar por algo, por la consecuencia de sus propias acciones. En otras palabras, la experiencia tiene que ver con lo que hacemos y sufrimos simultáneamente. (WEST, 2008, p. 102).

Esta concepción de experiencia dialoga con nuestra situación para poner en valor, en primer lugar, una dimensión o aspecto de la práctica política de autoorganización que no acude necesariamente a un registro cognitivo, que no se realiza externamente al mundo sino desde su padecimiento, desde su sufrimiento, desde algo que se vive con el cuerpo, desde el cuerpo en situación, y que a la vez imprime sus huellas en el cuerpo. Hay interacción, relación, continuidad, comunicación permanente e inmanente a la situación. Para las Madres, el poder de la experiencia consiste en 
primer lugar en haber vivido la situación desde dentro y no como mero espectador, tampoco como analista externo, sino desde un "estar ahí".

\section{Aprendizaje}

En paralelo a la petición a las autoridades, con sus propios recursos, madres y vecinos comienzan a elaborar el "mapa de la muerte": un dibujo de las manzanas y viviendas del barrio en el que identifican los enfermos, el tipo de patología, y las muertes. Las Madres hacen públicos los resultados del relevamiento, semana tras semana:

Llevamos registrados más de 200 casos de cáncer, sin contar Lupus, Púrpuras, Anemias Hemolíticas, Hodgkin Linfáticos, Tumores y Leucemias. Tenemos varios jóvenes fallecidos (22/23 años), uno de ellos murió a los tres días de su diagnóstico. Otros de 18 a 25 años tienen tumores en la cabeza; en el grupo de los de más corta edad hay cerca de 30 tumores (aún vive, pero están perdiendo, poco a poco, sus sentidos). Lo más terrible son los casos de leucemia ( 13 casos, tres de ellos están casa por medio y otros tres a un par de cuadras, entre dos transformadores y la plantación de soja que cubre el barrio). De estos 13 casos, las edades son:

-Niña de 5 años (vive) LLA

-Niña de 7 años (vive) LLA

-Niña de 13 años (vive) Leucemia Mixta

-Adolescente de 15 años (vive) LLA

-Adolescente de 17 años (vive) LLA-Adulto de 30 años (vive)

-Adulto de 50 años (vive) LLA

-Adulto de 57 años (fallecido) LLA

-Adulto de 23 años (fallecido) LLA

-Adulto de 30 años (Fallecido) LLA

-Matrimonio de 56-60 años (fallecidos) LLA

-Adulto de 58 años (vive)

Sabemos que solo entre dos a tres casos de Leucemia pueden esperarse en una población de 100,000 habitantes. En este año hemos tenido varios casos de malformación:

-Síndrome de Fryn (nacido con múltiples malformaciones, falleció al nacer)

-Espina Bífida (aún vive)

-Niño con 6 dedos (vive)

-Malformaciones de Riñón (vive)

-Osteogénesis (vive)

-Niña con múltiples malformaciones (falleció)

-Embarazada de 7 meses con niño con malformación (aún no ha nacido).

(GODOY et al., 2005). 
Es decir, la práctica de autoorganización genera saberes de tipo médico epidemiológico, conocimientos que los propios afectados producen ante el abandono estatal del cuidado de la salud pública. El conocimiento producido por los afectados es confirmado por los relevamientos epidemiológicos realizados por el Ministerio de Salud de la Provincia a partir del reclamo de las Madres. El primer informe oficial concluye que "la distribución de los afectados, al ser georeferenciada en el mapa del barrio, muestra un manchón epidemiológico, con 11 casos de pacientes con enfermedades oncológicas habitando en cuatro manzanas contiguas, lo cual motiva a continuar con las investigaciones", según establece un informe del Ministerio de Salud de la Provincia. Se realiza entonces un segundo relevamiento. Éste es específico sobre las manzanas donde se concentra el mayor número de casos, el "manchón epidemiológico" detectado. El instrumento se dirige a recabar casos de: patologías hematológicas (púrpuras, anemias), se encuentran 13 casos; linfomas, 6 casos; leucemia, 14 casos; cáncer (distintos tipos), 66 casos. En total se informan 109 casos de las patologías investigadas.

A partir de este diagnóstico, no sólo se confirman los casos denunciados por las Madres, sino que el gobierno provincial interviene con medidas asistenciales a partir de la presunta relación entre los contaminantes hallados y las enfermedades relevadas. Los resultados de los estudios sin embargo son cuestionados públicamente por las Madres, quienes acusan que no confirman todos los casos que van en aumento y que ellas continúan relevando e informando por su propia cuenta. El Ministerio instala en el barrio un servicio ambulatorio de oncólogos y psicólogos, destinados a hacer frente a la atención de las patologías relevadas y a "contener" las consecuencias psicológicas derivadas de las enfermedades.

Ante la gravedad de la situación epidemiológica y el aumento de las denuncias públicas, el Ministerio de salud contrata un servicio de consultoría de la Cátedra de Ingeniería Ambiental de la Universidad Nacional de Córdoba. Curiosa y preocupantemente, en sus conclusiones se lee: "No existen contaminantes ambientales que pudieran a priori afectar la salud de las personas, ya que se puede emitir juicio del factor agua y del factor atmósfera como habitables; en tanto que los factores contaminantes encontrados en suelo, atmósfera y agua, que habiéndose removido su fuente y no encontrado cantidades apreciables implican también habitabilidad". 
Esta denegación del problema por parte de las autoridades estatales lleva a las Madres a profundizar los aprendizajes de tipo organizativo, sus asambleas y métodos de protestaque ganan amplia visibilidad pública. Frente a ello, el gobierno despliega estrategias estatales intentan desarmar al grupo. A modo de ejemplo, el grupo fue invitado a formarse como promotores de salud por parte de un programa de salud estatal. Ellas estaban sosteniendo, como parte de su accionar, el relevamiento de las personas afectadas con todas las enfermedades relacionadas a la contaminación ambiental, con lo cual formulan sus denuncias y llevan adelante su pelea con el gobierno. Pero en el marco de la formación como promotoras de salud, la actividad corre el riesgo de ser reconvertida. "Ahora no sólo tenemos que ver los enfermos de cáncer sino que también tenemos que hacer captación temprana de pacientes hipertensos, de llevar a la gente al centro de salud", relata una de las integrantes del grupo.

Más allá de los efectivos procesos de aprendizaje, interesa señalar los efectos que tienen estas estrategias tanto de formación de promotores como la conformación de mesas de trabajo. Se desdibuja la identidad grupal, ya que traslada la referencia de la lucha, que era el colectivo de las Madres, a un espacio de trabajo conformado por varios actores de distintas procedencias y afiliaciones institucionales dentro de la comunidad. También como efecto se produce el descentramiento del colectivo de Madres respecto del objetivo de su lucha, la verdad y la justicia, entramadas ahora como tareas de gestión y burocráticas: el acompañamiento de pacientes para obtener medicamentos, expedientes y notas a los funcionarios públicos por temas relacionados a la problemática del barrio, transporte, pavimento, etc. ¿Cuáles son los temas sobre los que se puede deliberar y actuar efectivamente? ¿Cuáles son las posibilidades políticas de un espacio de participación basado en la autonomía ciudadana, en contextos de un estado que al momento no ha hecho justicia a la problemática del barrio? Algunas de las integrantes del grupo de Madres manifiestan su resistencia:

Quieren ponernos un freno, no quieren que seamos independientes, tratan de agarrarnos para ellos y lentamente nos van a ir desarmando [...] tenemos que pensar estas cosas. Antes hacíamos reuniones, convocábamos más gente, ahora no lo estamos haciendo más, y lo necesitamos.

Sumado a estas operaciones estatales que reorganizan la participación ciudadana, en el barrio las Madres se enfrenta a formas de violencia como la denegación de identidad, amenazas físicas, discriminación, descalificación de sus 
denuncias por parte de funcionarios de la administración estatal. Ante ello, la resistencia en la defensa de sus derechos, lleva a las Madres a la conformación de una red de actores a nivel provincial, nacional y transnacional. De esta forma, la lucha solidaria con otras organizaciones en contra la contaminación y la fumigación con agrotóxicos constituye una nueva etapa de la lucha de las Madres y un modo de sostener la denuncia a pesar de que a nivel local se deniegue la problemática. La campaña nacional Paren de Fumigar, así como la participación de las Madres en la Cumbre Alternativa de la Salud de los Pueblos y otros eventos internacionales muestran el aprendizaje de un accionar concertado en una causa común, y trascendiendo las fronteras locales.

Con estos y otros ejemplos, nos interesa el significado de la experiencia como aprendizaje en tanto que saber hacer. El conocimiento se produce como actividad de lucha, no como intelección separada del contexto de la acción. Que el conocimiento sea considerado como acción significa poder pensar que en la experiencia de las Madres no hay distinción entre conocedor y hacedor. Frente a la denegación de sus capacidades por parte de funcionarios públicos, frente al cuestionamiento de su estrategia en la red de actores comprometidos en su lucha, las Madres acreditan con su acción el aprendizaje. Es en la lucha donde se adquieren nuevos lenguajes, sean técnicos (legales, médicos, ambientales) y políticos (de autoorganización, de interacción con los funcionarios, de aparición en el espacio público). La cognición "encarnada" es el resultado de un proceso de variación, cambio, selección, situado en una dinámica de continuidad entre el organismo y su ambiente, centrada en el problema, opera en relación a las necesidades, intereses y valores de los organismos, no procurando la solución perfecta sino una que trabaje lo suficientemente bien para una situación corriente, que se lleva a cabo intercorporal y cooperativamente. La acumulación de estos intercambios e interacciones a nivel social es lo que nos permite pensar la experiencia como aprendizaje.

\section{Experiencia / experimentar}

Continuando con los aportes de Dewey para pensar la noción de experiencia, el autor argumenta que el hombre no es un espectador mirando desde afuera y que simplemente recibe y registra percepciones pasadas. El hombre es un agente, un experimentador, es esencialmente una creatura orientada al futuro. Para entender la experiencia humana tenemos que tener en mente que el hombre es agente 
(BERNSTEIN, 1996, p. 67) que siempre vive hacia adelante. Dewey acentúa el futuro, el sentido prospectivo de la experiencia humana, siguiendo el giro pragmatista que se separa de las verdades evidentes y las fundaciones epistémicas. La experiencia es un futuro como implicación de un presente, exclama Dewey (WEST, 2008). La experiencia es experimental:

[...] Las cosas que vivimos son experimentos para modificar el curso de los acontecimientos, nuestros intentos activos son pruebas y ensayos de nosotros mismos...Nada puede eliminar todo el riesgo, toda la aventura. Los obstáculos a los que nos enfrentamos son estímulos para la variación, para respuestas novedosas y son, por tanto, ocasiones para el progreso. (WEST, 2008, p. 103).

Recordando que la situación de la acción de las Madres es la de la interperie y el abandono de estructuras institucionales de reconocimiento y protección de los derechos. Las Madres se encuentran luchando solas junto a otros afectados por las fumigaciones con agrotóxicos, ante el abandono también de actores tradicionales de la política y formas instituídas para la resolución de los problemas públicas. La crisis, la decadencia o la ausencia de estos componentes, la experiencia se puede comprender como la creatividad de formas de actuar democráticas, en defensa de lo público- común.

Entre los logros que han obtenido las Madres con su perseverancia, con la fuerza de su autoorganización y con la solidaridad de una red de actores, podemos mencionar la promulgación de leyes provinciales y ordenanzas municipales que regulan la fumigación con agrotóxicos; medidas asistenciales del estado creando centros de salud municipal y áreas de epidemiología ambiental en el ministerio de salud de la provincia; protocolos de intervención para poblaciones expuestas agrotóxicos por parte del gobierno nacional.

Sin embargo y pese a estas conquistas, el problema continúa, ya que las autoridades estatales de todos los niveles de gobierno (municipal, provincial y nacional) no reconocen públicamente el problema, no han intervenido en la reparación del daño ni el resarcimiento a las víctimas, que siguen desatendidas y buscando individualmente la solución para sus enfermedades. La regulación de las fumigaciones no se realiza por falta de controles estatales, y la administración de justicia tampoco ha avanzado en sancionar los delitos ambientales cometidos.

Esta situación de cuasi abandono institucional exige de los ciudadanos una permanente revisión de su curso de acción, y el ensayo de otras modalidades de protesta. Por ello nos interesa pensar en términos pragmatistas sobre la creatividad 
de la acción. Para Joas (1996, 1998), por ejemplo, la metáfora directriz de la acción no es ni la expresión poética, ni la producción material ni la transformación revolucionaria de la sociedad, sino la resolución creativa de los problemas por parte de una inteligencia que experimenta.

En el caso de los problemas de la acción, se establecen experimentalmente conexiones entre los impulsos y las posibilidades de acción [...] el curso de acción no se establece de una vez por todas, lo típico es la revisión continua y la constante generación del curso de acción que sigue. (JOAS, 1998, p. 283).

\section{Narración}

En el caso nuestro, se han violado los derechos humanos y lo que nosotros estamos pidiendo es un derecho a la salud, a la vida y a un medio ambiente sano, que son prácticamente, si vos te das cuenta, un derecho que tendríamos que tener todos y ahora no lo tenemos. Es algo intransferible el derecho a la vida, y violar ese derecho, que es un derecho humano, hace mal a todo; han pisoteado lo más sagrado de una persona.

La lucha de las Madres enuncia el discurso de los derechos. Saben como cualquier ciudadano que pueden enunciar/ invocar los derechos a la vida y a la salud, constitucionalmente reconocidos ante la violación de los mismos y dirigir su reclamo al gobierno. Saben también que hay una forma de hacerlo, instituída por fuera de la constitución y las leyes: "Uno empieza como se empezó en la Plaza de Mayo el 20 de diciembre; la gente sabía que tenía que salir a la calle a reclamar y pasó eso...”, como relatan las Madres. La institucionalidad democrática de la calle y la asamblea, que en pleno 2001 expresan vastos sectores de la población, personas y colectivos que defienden sus derechos apelando a sus propias fuerzas y capacidades ante el abandono institucional.

En cuanto a la creatividad de la acción a la que hacíamos referencia en el apartado anterior, ésta también puede ser entendida como la generación de marcos y palabras para sus denuncias públicas, como el uso de genocidio.

Seguimos sosteniendo que la situación que vivimos en el barrio constituye un genocidio encubierto, porque estamos finalizando el 2006 y los intereses económicos continúan estando por encima de la salud de la población, ya que tenemos niños con análisis que verifican que tienen agroquímicos en la sangre, lo que demuestra que nuestras denuncias no son producto de nuestra imaginación. Ante esta situación, las autoridades competentes siguen negando la realidad y por lo tanto la solución. Contamos con todas las pruebas científicas de que la problemática del barrio es la alta contaminación ambiental [...] y a pesar de ello la justicia no hace lugar a las querellas presentadas por nuestra abogada. A ello hay que agregar que la situación del barrio sigue agravándose, ahora no se trata de un número sino de toda una población. 
Si consideramos que el significado de las palabras y el sentido de las oraciones están dados por su uso, nuestra posición frente a este uso de genocidio ambiental no fue una evaluación acerca de su corrección o pertinencia, sino indagar acerca de la gramática que envuelve este uso, es decir que lo posibilita o restringe. El uso del concepto se da en un contexto en el que se señala que las autoridades no cumplen con su función de velar por la salud de la población, a pesar de la existencia de derechos reconocidos constitucionalmente y legislación específica. Ahora bien, en esta institucionalidad, más cerca de la figura del estado de excepción que de la del democrático de derecho, las responsabilidades no se extinguen, y desde aquí es invocada la acción del estado como garante de derechos y evocado el concepto de genocidio como negación de los mismos por parte del estado (CARRIZO; BERGER, 2009, p. 272).

Si junto a este uso de genocidio agregamos que la identidad de Madres en la denominación del colectivo evoca la identidad de las Madres de Plaza de Mayo en la última dictadura militar en Argentina, podemos señalar que hay una construcción narrativa que asigna un sentido a la lucha por la vida, una actualidad de los derechos humanos.

En la época que iba a ser el mundial, las Madres empezaron a hacer las denuncias, solas, dando vueltas a Plaza de Mayo, ¿qué decía la gente?: ‘¡si desaparecieron por algo será!’, ‘¡algo habrán hecho!’. Sí, seguramente habían salido en defensa de sus derechos y había que callarlas de alguna manera, y a nosotros no nos han callado de esa forma drástica, que directamente te tiraban al río, a nosotros nos van callando de otra manera.

Los aportes de Ricoeur contribuyen a comprender las construcciones identitarias con el paradigma textual, el agente es a la acción lo que el autor al texto. El significado del texto escapa al autor y se plasma en la interpretación en el espacio público. Como señala el autor:

La noción de texto es un buen paradigma para la acción humana y, por la otra, que la acción es un buen referente para toda una categoría de textos [...] la acción humana está exteriorizada de una manera comparable a la fijación característica de la escritura. Al desprenderse de su agente, la acción adquiere una autonomía semejante a la autonomía del texto, deja un trazo, una marca, se inscribe en el curso de las cosas y se vuelve un archivo, un documento (RICOEUR, 1988, p. 87).

La apropiación que realizamos del giro narrativo se vincula con la posibilidad de apropiación de la experiencia por parte de los protagonistas, una narración, un sentido para la lucha. Si consideramos entonces el texto como acción, la fuerza de la práctica de la autoorganización también se encuentra en el poder decir 
que implica la capacidad narrativa de los ciudadanos que defienden derechos. En la situación de las Madres de Barrio Ituzaingó Anexo, este poder resiste a las operaciones de denegación de identidad, cuando las autoridades públicas desconocen la problemática del barrio o actúan de forma deficitaria en la solución del mismo, las Madres siguen denunciando genocidio encubierto, como testimonio del dolor y la injusticia que padecen los afectados a casi diez años de iniciada la lucha por los derechos vulnerados.

No es contradictorio sino paradojal del ejercicio de los derechos en las democracias realmente existentes, el dirigir los reclamos por derechos al estado que es parte responsable por la vulneración de los mismos. En la defensa de la vida se afirma el valor de la misma, y se reafirma la posibilidad de la política no contra o sobre la vida sino a favor de ella. Siguiendo a Ricoeur, la figura de la paradoja nos permite pensar que en el caso de la defensa de los derechos, contra la vulneración hay resistencia. La autonomía es tanto una declaración como un proyecto, una tarea política frente a su cuestionamiento. Al decir del mencionado autor:

Es el mismo ser humano el que es lo uno y lo otro bajo dos puntos de vista diferentes. Y es más, no contentos con oponerse, los dos términos se componen entre sí: la autonomía es la de un ser frágil, vulnerable. Y la fragilidad no sería más que una patología, sino fuera por la fragilidad de un ser llamado a llegar a ser autónomo, porque lo es desde siempre de una cierta manera. (RICOEUR, 2008, p. 71).

\section{A modo de conclusiones}

Nos interesa pensar la actualidad de la defensa de derechos en casos de contaminación ambiental por agrotóxicos. La lucha de las Madres de Barrio Ituzaingó, que hemos presentado muy brevemente ha contribuído a nuestras reflexiones. Preocupados por los logros de su acción y los problemas que ésta enfrenta, como investigadores de una universidad pública democrática no podemos menos que compartir con ellas el sentido de justicia en la lucha por la vida y la salud. La práctica de investigación es entendida junto a Pitkin como la posibilidad de restituir la función reveladora de verdad de nuestro lenguaje y de su poder para establecer relaciones genuinas con nuestros conciudadanos (PITKIN, 1984).

Vemos que en el contexto del estado democrático de derecho, son las prácticas ciudadanas las que defienden lo público de la política a través de su acción, y no las estructuras institucionales del estado, supuestas garantes de los derechos. En esta situación, el único modo de práctico de observar el poder es en su ejercicio, 
siempre relacionalmente, es decir, que los fenómenos de poder ocurren entre la gente, y que las relaciones de poder pueden alterarse. Por ello, hemos identificado en las nociones de cuerpo, experiencia y narración aspectos del poder de hacer y decir que se anudan en la práctica ciudadana, en realidades tan difíciles como las que actualmente continúan enfrentando las Madres de Barrio Ituzaingó Anexo.

\section{Referencias}

AGAMBEN, Giorgio. Homo Sacer. El poder soberano y la nuda vida I. Valencia: Pre-Textos, 1998.

. Medios sin fin. Valencia: Pre-textos, 2001.

. “QQué es un dispositivo?”. Conferencia pronunciada en la Universidad Nacional de la Plata. La Plata, octubre 2005.

ARENDT, Ana. De la historia a la acción. Barcelona: Paidós, 1995.

- ¿Qué es la política? Barcelona: Paidós, 2001.

BERNSTEIN, Richard. John Dewey. Ohio: Ridgeview Publishing Company, 1996.

CARRIZO, Cecilia; BERGER, Mauricio: Estado incivil y ciudadanos sin estado. Paradojas del ejercicio de derechos en cuestiones ambientales. Córdoba: Narvaja, 2009.

DEWEY, John. La opinión pública y sus problemas. Estudio preliminar y revisión por Ramón del Castillo. Madrid: Morata, 2004.

EDER, Klaus. La institucionalización de la acción colectiva. ¿Hacia una nueva problemática teórica en el análisis de los Movimientos Sociales? In: IBARRA, Pedro; TEJERINA, Benjamín (Ed.). Los Movimientos Sociales. Transformaciones políticas y cambio cultural. Madrid: Editorial Trotta, 1998.

FOUCAULT, Michel. Genealogía del racismo. La Plata: Altamira, 1996.

. Historia de la sexualidad. Tomo 1. La voluntad del saber. Buenos Aires: Siglo Veintiuno, 2002.

2006.

- Seguridad, territorio, población. Buenos Aires: Fondo de Cultura Económica,

GODOY, María et al. Destrucción del Espacio Urbano: Genocidio Encubierto en Barrio Ituzaingó Anexo de Córdoba. En Informe Alternativo sobre la Salud en América Latina. Observatorio Latinoamericano de Salud. Ecuador: CEAS, 2005.

GOFFMAN, Irving. Frame Analysis. Los marcos de la experiencia. Madrid: CIS, 2006.

JOAS, Hans. El pragmatismo y la teoría de la sociedad. Madrid: CIS/SXXI, 1998.

. The Creativity of action. Chicago: The University of Chicago Press, 1996.

NAISHTAT, Francisco. Problemas filosóficos en la acción individual y colectiva: una perspecti- 
va pragmática. Buenos Aires: Prometeo Libros, 2005.

NEGRI, Antonio; HARDT, Michael. Imperio. Buenos Aires: Paidós, 2001.

. Multitud. Guerra y democracia en la era del Imperio. Buenos Aires: Editorial Debate, 2004.

ORTEGA, Francisco. La abstracta desnudez de ser únicamente humano. Racismo y biopolítica en Hannah Arendt y Michel Foucault. In: UGARTE PÉREZ, Javier (Org.). La administración de la vida. Estudios biopolíticos. Barcelona: Anthropos, 2005.

. Reflexões a partir de Michel Foucault, Agnes Heller e Hannah Arendt. Interface: Comunic., Saúde, Educ., v. 8, n. 14, p. 9-20, set. 2003-fev. 2004.

PITKIN, Hannah. Wittgenstein. El lenguaje, la política y la justicia. Madrid: Centro de Estudios Constitucionales, 1984.

RICOEUR, Paul. Lo Justo 2. Estudios, lecturas y ejercicios de ética aplicada. Madrid: Editorial Trotta, 2008.

. Historia y narratividad. Barcelona: Paidós, 1999.

. Hermenéutica y acción. Buenos Aires: Editorial Docencia, 1988.

VIRNO, Paolo. Gramática de la Multitud. Para un análisis de las formas de vida contemporáneas. Buenos Aires: Editorial Colihue, 2003.

. Cuando el verbo se hace carne. Lenguaje y Naturaleza Humana. Buenos Aires: Cactus, Tinta Limón, 2005.

WEISS, Gail. Body Images. Embodiment as intercorporeality. New Cork: Routeledge, 1999.

WEST, Cornel. La evasión americana de la filosofía. Una genealogía del pragmatismo. Madrid: Complutense, 2008.

\section{Notas}

${ }^{1}$ Este artículo fue realizado en el marco de las misiones académicas entre el Doctorado en Ciencias Sociales de la Universidad de Buenos Aires, Argentina y el Instituto de Medicina Social de la Universidad Estadual de Rio de Janeiro, Brasil. Mi agradecimiento a los responsables de las mismas, y especialmente a los integrantes del Programa de Estudios en Acción y Sujeto del Instituto de Medicina Socual de la Universidade Estadual do Rio de Janeiro por su orientación académica y la amable recepción de mi estadía de investigación.

${ }^{2}$ Los testimonios de las Madres presentes en este artículo provienen de conversaciones con las integrantes del colectivo, materiales producidos en nuestra investigación doctoral y principalemente por el Proyecto Ciudadanía, en el Instituto de Investigación y Formación en Administración Pública, Universidad Nacional de Córdoba, Argentina. Agradezco a la Prof. Mgter. Cecilia Carrizo la permanente orientación académica en mi formación doctoral. 


\section{Abstract}

Populations exposed to agrotoxics: citizens'selforganization in the defense of life and health, Córdoba City, Argentina

This paper presents a preview of our doctoral research work, based in the political action of the groups selfdenominated "Madres de Barrio Ituzaingó Anexo" in the city of Córdoba, Argentina. Since late 2001, the members of this group started fighting for life, health and environment, in the context of an environmental problem that produces over 200 illnesses and deaths in a population of 5,000 habitants in the neigborghood, mainly caused by fumigations with agrotoxics in the soybean fields adjacent to the houses. The modes of self-organization displayed by "las Madres" against the infringement of their rights plus the inefficiency and also abandonment of the State in the protection of the ones affected by the pollution, made this citizenship practice an example to think about the struggle for rights. This paper is divided into three sections. First, we present the methodological and conceptual elections that frame our reflections: the contributions of pragmatism (Dewey and Joas), the narrative turn (Ricoeur) and contemporary discussions in biopolitics (Agamben, Hardt y Negri, Virno). Second, we join the fight of the Madres de Barrio Ituzaingó Anexo and their contexts. Third, we describe some scenes of the fight of the Madres against the fumigation with agrotoxics, and to that purpose we introduce three notions to think the citizen self-organization: body, experience, narrative. These will be considered key aspects of the political practice in which the sense of politics as action is played, as the power of making and speaking of the citizenship that acts in self-defense. Through the act of their affective, cognitive and linguistic capacities, these citizens create learnings, practical wisdoms and achievements in the defense of their rights, but they are not free from problems and obstacles in their claim for justice.

> Key words: action; rights; health; environment; agrotoxics. 